\title{
The Application of Cauchy Inequality in Middle School Mathematics
}

\author{
Ouyang Yun, ${ }^{a}$ Zedong Lai ${ }^{b}$, Wusheng Wang ${ }^{c}$
}

School of Mathematics and Statistics, Hechi University, Guangxi, Yizhou 546300, P. R. China

as huxueoyy@126.com, b18776832937@126.com, cwang4896@126.com

Keywords: Cauchy Inequality; Proof of Inequality ; Extreme Value Problems; Solution Procedure.

\begin{abstract}
Cauchy inequality is widely applied in the middle school mathematics. This paper first studies the Cauchy inequality, then discusses the application skills of Cauchy inequality in the middle school mathematics, illustrates applications of the Cauchy inequality in the proof of the complex inequalities and the extreme value problems, and finally summarizes the application scope of Cauchy inequality and solution procedure.
\end{abstract}

\section{Introduction}

Cauchy inequality is widely applied in the middle school mathematics. Many authors[1-9] studied the problems in the middle school mathematics by using Cauchy inequality. Cauchy inequality can be stated as follows:

Suppose that $a_{1}, a_{2}, \cdots, a_{n} ; b_{1}, b_{2}, \cdots, b_{n}$ are two groups of real numbers, then inequality

$$
\sum_{k=1}^{n} a_{k}^{2} \sum_{k=1}^{n} b_{k}^{2} \geq\left(\sum_{k=1}^{n} a_{k} b_{k}\right)^{2},
$$

holds. Equality holds when and only when $a_{1}: a_{2}: \cdots: a_{n}=b_{1}: b_{2}: \cdots: b_{n}$.

Cai [3] discussed the various equivalent forms of the Cauchy inequality.

Let $a_{1}, a_{2}, \cdots, a_{n}$ are positive real numbers, then

$$
\left(a_{1}+a_{2}+\cdots+a_{n}\right)\left(\frac{1}{a_{1}}+\frac{1}{a_{2}}+\cdots+\frac{1}{a_{n}}\right) \geq n^{2} .
$$

Equality holds when and only when $a_{1}=a_{2}=\cdots=a_{n}$.

Let $a_{1}, a_{2}, \cdots, a_{n}$ are real numbers, then

$$
n \sum_{k=1}^{n} a_{k}^{2} \geq\left(\sum_{k=1}^{n} a_{k}\right)^{2} \text {. }
$$

Equality holds when and only when $a_{1}=a_{2}=\cdots=a_{n}$.

In (3), if $a_{1}, a_{2}, \cdots, a_{n}$ are positive real numbers, then Cauchy inequality has the following equivalent forms:

$$
\begin{aligned}
\sum_{k=1}^{n} a_{k} b_{k} \sum_{k=1}^{n} \frac{a_{k}}{b_{k}} \geq\left(\sum_{k=1}^{n} a_{k}\right)^{2}, \\
\sum_{k=1}^{n} b_{k} \sum_{k=1}^{n} \frac{a_{k}^{2}}{b_{k}} \geq\left(\sum_{k=1}^{n} a_{k}\right)^{2},
\end{aligned}
$$




$$
\sqrt{\sum_{k=1}^{n} a_{k} \sum_{k=1}^{n} b_{k}} \geq \sum_{k=1}^{n} \sqrt{a_{k} b_{k}}
$$

\section{Application of Cauchy Inequality in Middle School Mathematics}

Using the condition of equality holding in Cauchy inequality, we prove identical equations.

Example 1 If $a \sqrt{1-b^{2}}+b \sqrt{1-a^{2}}=1$, then $a^{2}+b^{2}=1$.

Proof. By Cauchy inequality, we have

$$
a \sqrt{1-b^{2}}+b \sqrt{1-a^{2}} \leq\left[a^{2}+\left(1-a^{2}\right)\right]\left[\left(1-b^{2}\right)+b^{2}\right]=1
$$

Since $a \sqrt{1-b^{2}}+b \sqrt{1-a^{2}}=1$, we have

$$
\left.a \sqrt{1-b^{2}}+b \sqrt{1-a^{2}}=\left[a^{2}+\left(1+a^{2}\right)\right]\left(1-b^{2}\right)+b^{2}\right] .
$$

So $\frac{\sqrt{1-b^{2}}}{a}=\frac{b}{\sqrt{1-a^{2}}}, a^{2}+b^{2}=1$.

Proving complex inequalities using Cauchy inequality

Example 2[3] If $a, b, c, d$, are positive real numbers, and $a+b+c+d=4$, then

$$
\frac{a^{2}}{b}+\frac{b^{2}}{c}+\frac{c^{2}}{d}+\frac{d^{2}}{a} \geq 4+(a-b)^{2} .
$$

Proof. Since

$$
\begin{aligned}
& \frac{a^{2}}{b}+\frac{b^{2}}{c}+\frac{c^{2}}{d}+\frac{d^{2}}{a}-(a+b+c+d) \\
= & \left(\frac{a^{2}}{b}+b-2 a\right)+\left(\frac{b^{2}}{c}+c-2 b\right)+\left(\frac{c^{2}}{d}+d-2 c\right)+\left(\frac{d^{2}}{a}+a-2 d\right) \\
= & \frac{(a-b)^{2}}{b}+\frac{(b-c)^{2}}{c}+\frac{(c-d)^{2}}{d}+\frac{(d-a)^{2}}{a} .
\end{aligned}
$$

From (5) we get

$$
\begin{gathered}
{\left[\frac{(a-b)^{2}}{b}+\frac{(b-c)^{2}}{c}+\frac{(c-d)^{2}}{d}+\frac{(d-a)^{2}}{a}\right](a+b+c+d)} \\
\geq(|a-b|+|b-c|+|c-d|+|d-a|)^{2} .
\end{gathered}
$$

Since $|b-c|+|c-d|+|d-a| \geq|a-b|$, we have

$$
(|a-b|+|b-c|+|c-d|+|d-a|)^{2} \geq 4(a-b)^{2}
$$

From (8), (9) and (10),we have

$$
\frac{a^{2}}{b}+\frac{b^{2}}{c}+\frac{c^{2}}{d}+\frac{d^{2}}{a} \geq a+b+c+d+\frac{4(a-b)^{2}}{a+b+c+d} .
$$


Since $a+b+c+d=4$, From (11), we have

$$
\frac{a^{2}}{b}+\frac{b^{2}}{c}+\frac{c^{2}}{d}+\frac{d^{2}}{a} \geq 4+(a-b)^{2} .
$$

Finding extreme value problems by using Cauchy inequality

Example 3[1] Analy se the maximum of $y=3 \sqrt{x-5}+4 \sqrt{9-x}$.

using Cauchy inequality $\left|a_{1} b_{1}+a_{2} b_{2}\right| \leq \sqrt{\left(a_{1}^{2}+a_{2}^{2}\right)\left(b_{1}^{2}+b_{2}^{2}\right)}$, we get

$$
3 \sqrt{x-5}+4 \sqrt{9-x} \leq \sqrt{\left(3^{2}+4^{2}\right)\left[(\sqrt{x-5})^{2}+(\sqrt{9-x})^{2}\right]}=10 .
$$

Solving equation using Cauchy inequality

Example 4[3] Solve the equation

$$
\sqrt{x-\frac{1}{x}}+\sqrt{1-\frac{1}{x}}=x, x>1 .
$$

In order to using Cauchy inequality, we construct two groups of real numbers

$$
\sqrt{x-\frac{1}{x}}, \sqrt{\frac{1}{x}}, 1, \sqrt{x-1} .
$$

Using Cauchy inequality (1), we obtain

$$
\begin{gathered}
\left(\sqrt{x-\frac{1}{x}}+\sqrt{1-\frac{1}{x}}\right)^{2}=\left(\sqrt{x-\frac{1}{x}}+\sqrt{\frac{1}{x}} \cdot \sqrt{x-1}\right)^{2} \\
\leq\left(x-\frac{1}{x}+\frac{1}{x}\right)(1+x-1)=x^{2} .
\end{gathered}
$$

Since $\sqrt{x-\frac{1}{x}}+\sqrt{1-\frac{1}{x}}=x$, we have

$$
\left(\sqrt{x-\frac{1}{x}}+\sqrt{\frac{1}{x}} \cdot \sqrt{x-1}\right)^{2}=\left(x-\frac{1}{x}+\frac{1}{x}\right)(1+x-1) .
$$

Using Cauchy inequality (1), we have

$\sqrt{x-\frac{1}{x}} \cdot \sqrt{x-1}=\sqrt{\frac{1}{x}} \Leftrightarrow x\left(x^{2}-x-1\right)=0 \Leftrightarrow x=\frac{1+\sqrt{5}}{2}$.

After checking, we see that $x=\frac{1+\sqrt{5}}{2}$ is the root of equation (12).

\section{Summary}

This paper studies the Cauchy inequality, discusses the application skills of Cauchy inequality in the middle school mathematics, illustrates the applications of the Cauchy inequality in the proof of the complex inequalities and the extreme value problems, and finally summarizes the application scope of Cauchy inequality and solution procedure. 


\section{Acknowledgement}

This research was supported by Guangxi Natural Science Foundation (Project No. 2012GXNS FAA053009) and Scientific Research Foundation of the Education Department of Guangxi Autonomous Region of China (No. KY2015ZD103), and the high school specialty and curriculum integration project of Guangxi Zhuang Autonomous Region (No.GXTSZY2220).

\section{References}

[1] X.Chen,Y. Liu. Elementary Mathematics Research[M]. Beijing: Peking University Press. 2012:64-66.

[2] S.Chen. Cauchy Inequality and the 2008 College Entrance Exam [J]. Middle School Mathematics. 3(2009)(3)21-22.

[3] Y.Cai. Proving Methods and Skills of Inequality in Mathematical Olympiad [M]. Harbin Institute of Technology Press. 2011:196-226.

[4] J.Li, H. Li. Using Cauchy Inequality for Function Extremum [J]. Hebei Energy Institute of Vocational and Technology. 1(2002)94-96.

[5] Q. Li. The Proof and Application of Cauchy Inequality in Middle School Mathematics [J]. Jinggangshan College of Science.29 (2008)124-125.

6] X. Yu. The Status Quo and Strategy of Cauchy Inequality Teaching [J]. Mathematics Bulletin, 6(2014)36-43.

[7] Z.Chen. The Proof of Cauchy Inequality and Its Application in Extremum Problem [J]. Examination weekly, 85(2008)73-74.

[8] L.Wang. The Utilization of Cauchy Inequality in Elementary Mathematics[J]. China Education Innovation Herald, 26(2008)148-150. 